\title{
Specific impairments and predictive markers for developmental coordination disorder subtypes in children: The importance of multidimensional developmental
} assessments in cluster analysis

\author{
Vaivre-Douret $\mathrm{L}^{1,2,3,4,5,6 *}$ and Lalanne $\mathrm{C}^{7}$ \\ ${ }^{1}$ Faculty of Medicine, University of Paris, Paris-Descartes, Sorbonne Paris City (SPC), Paris, France \\ ${ }^{2}$ INSERM Unit 1178/1018-CESP, University of Paris Sud-Paris Saclay, UVSQVillejuif and Paris Descartes, SPC, Necker-Enfants-Malades University Hospital, \\ AP-HP, Paris, France \\ ${ }^{3}$ Department of Child Psychiatry, Necker-Enfants Malades University Hospital, AP-HP, Paris, France \\ ${ }^{4}$ Department of Pediatrics, AP-HP Paris Center Port Royal-Cochin Hospital, Paris, France \\ ${ }^{5}$ Hospitalo University Institute Imagine, Paris, France \\ ${ }^{6}$ University Institut of France (IUF), Paris, France \\ ${ }^{7}$ Patient-Centered Outcomes Research, Sorbonne Paris City, EA 7334 (REMES), University of Paris, Diderot and Saint-Louis Hospital, Paris, France
}

\begin{abstract}
Developmental Coordination Disorder (DCD) defines a heterogeneous group of children exhibiting impairments in motor coordination that significantly interferes with an individual's academic achievement and/or activities of daily living. No concensus and little interest are noticed in the literature about researches of DCD subtypes due to methodology, while it could provide an important contribution to an understanding of DCD (semiology, etiology, neurophysiological brain mecanisms) to better define a therapeutic orientation. Only some studies have used cluster analysis as a method to identify distinct subtypes of children with DCD. In this approach a set of measurements is acquired and subjects are grouped together on the basis of the profiles of their scores. However, few studies have investigated different cerebral functions alongside a standardized motor developmental assessment. We will report and analyse the characteristics of DCD populations in studies and features of subtypes in clusters available in the literature and we will focus on model studies using strict inclusion criteria of participants with a multidimentional investigations composed of standardized psychological, neuropsychological, developmental neuromotor and psychomotor assessments, accompanied by neurovisual examination. Multivariate supervised statistical models were used to isolate specific markers of three subtypes of DCD (Ideomotor, Visuo-Spatial and Constructional, and the Mixed subgroup) with high predictive discriminant power on an augmented database of DCD children. The findings suggest that a set of appropriate measures of body structure and cognitive functions should be provided for DCD diagnosis and subtyping. The most discriminant markers (significant, $\mathrm{p}<0.05$ ) to explain the specificity of the three subtypes evidenced are: digital praxis, imitation of gestures, digital perception, visual motor integration, manual dexterity, visual spatial structuration, coordination between upper and lower limbs, and Lego blocks. This was confirmed by two multivariate statistical models (100\% with Random Forest (RF) and 91,4 \%, (0.713-0.999) with PLS discriminant analysis). Investigations of motor coordination should use a standardized neurodevelopmental assessment (with age-related normative data) with identical independent subtests across ages and qualitative and quantitative measures (such as NP-Mot Battery) reflecting the development of neuro-physiological mechanisms in child that contribute to movement timing, motor control, motor coordinationand motor execution. It is completed by cognitive and neurovisual investigations. Performance test with global score such as the Movement Assessment Battery for Children (M-ABC), the most used in cluster studies and researches on DCD is questionable to diagnose DCD. Implications for the understanding of the nature and neurophysiological brain mechanisms involved in subtyping DCD are discussed with regard to multidimensional standardized investigations. Further researches in DCD subtyping may be encouraged for a better orientation of remediation in clinical practice.
\end{abstract}

\section{Introduction}

The interest in Developmental Coordination Disorder (DCD) has grown considerably over recent years. DCD is characterized by marked impairment in motor coordination, fine and/or global, that significantly interferes with an individual's academic achievement and/ or activities of daily living (criterion B), not explained by intellectual disability, visual impairment, or neurological deficit such as weakness or cerebral palsy, muscular dystrophy, etc (criterion D of the DSM-5 criteria in Diagnostic and Statistical Manual of Mental Disorders), [1]. Maturational delay is also observed in the acquisition or in execution of coordinated motor skills (criterion A) interfering with the child's academic achievement and activities of daily living, leisure, and play (criterion B).

${ }^{\star}$ Correspondence to: Professor Laurence Vaivre-Douret, Hopital Universitaire Necker-Enfants Malades, INSERM UMR 1178/1018 CESP, Carre Necker, Porte N4, 149 rue de Sèvres, 75015 Paris, France, E-mail: laurence.vaivre-douret@inserm.fr

Key words: developmental coordination disorder, subtypes of dcd, developmental assessment, motor impairment, soft-signs, visual-spatial motor impairment, diagnostic markers, neurovisual, co-morbidities, clusters, multivariate analysis

Received: April 19, 2019; Accepted: May 13, 2019; Published: May 16, 2019 
Vaivre-Douret L (2019) Specific impairments and predictive markers for developmental coordination disorder subtypes in children: The importance of multidimensional developmental assessments in cluster analysis

DCD is an idiopathic disorder which affects approximately 5-6\% of school-age children, with a higher incidence among boys than girls, symptoms of DCD have to be present in early childhood (criterion C of the DSM-5) [1]. In the literature, DCD appears across a heterogeneous characteristics of children, with different features of the sensory-motor and perceptual-motor processing components of movement [2-12]. Nevertheless, no consensus has been reached either on a common aetiology or on the identification of the nature of DCD. Few researchers have used cluster analysis to identify distinct subtypes of children with DCD and the existing cluster studies show little consistency (for a review, see Macnab, Miller, \& Polatajko, 2001 [8]; Vaivre-Douret, 2014 [13]) because they do not use the same measures, often just one motor test is achieved, and sampling information or detailed characteristics of the participants are often lacking (e.g. birth term, remediation, drugs, co-morbidity of neurodevelopmental disorders...). Thus, no clear consensus on the subtyping of developmental coordination disorder has been reached [11].

In the current article, we report and analyse in a first part, the characteristics of DCD populations in studies and features of subtypes in clusters available in the literature. Secondarily, we will focus on model studies using strict inclusion criteria of participants with a multidimentional investigations composed of standardized psychological, neuropsychological, developmental neuromotor and psychomotor assessments, accompanied by neurovisual examination, permitting to distinguish three main subtypes of DCD. In addition, we will present the results of a second study using multivariate statistical models making it possible to isolate specific markers for each subtype with high predictive, discriminant power. We will discuss the nature of these specific deficits in the spectrum of motor disorders in DCD, defined as heterogeneous groups in the few existing DCD subtyping studies.

\section{Analysis of cluster studies in literature defining DCD subtypes}

Of nine main studies on subtypes of DCD [2,4-9,11,14], eight used performance results for motor skills from standardized motor assessments (norm-referenced) such as the Bruininks Oseretsky Test of Motor Proficiency (BOTMP) [15], or the Movement Assessment Battery for Children (M-ABC) [16]. Seven of these studies also used an augmented battery including neuropsychological assessments consisting in visual perceptual measures and/or visual motor integration with the VMI test $[2-5,8-9,11,17]$ and kinaesthetic perception $[2,5,8-9,11]$ or other cognitive assessments such as planning, attention, and simultaneous coding scales [14]. Three studies $[4,9,11]$ also used transitive gestures with motor sequencing. However, only Vaivre-Douret et al.[11-12] used a standardized developmental assessment (with identical subtests across ages) with quantitative and qualitative measures for every neuropsychomotor functions, alongside a neurological normed examination highlighting neurological soft signs [18]. In addition, these authors performed an in-depth neuropsychological evaluation of cerebral functions (subtests described in the previous study $[11,12,19]$ : the Wechsler measure of intelligence, tests of attention, mental executive functions, visual perception, visual motor perception, visual motor integration, kinaesthetic perception, memory, language, and electro-physiological visual function.

In all 9 studies on subtypes cited above, Ward-based hierarchical classification or the k-means algorithm were used to cluster subjects in homogeneous classes. However, the number of subtypes found remains heterogeneous across the nine studies because the data concerning the DCD children were imprecise with respect to the presence or absence of co-morbidities, numbers and characteristics, and it is not clear whether the information was known and not reported, or not assessed in the sample. Minor neurological dysfunctions or neurological soft signs for their part were only systematically explored in the study by Vaivre-Douret et al.[11,12,20]. Thus, the choice of measures has a considerable impact in determining the specific features in each group, and in understanding the nature, aetiology and mechanisms of DCD subtypes. It is not surprising that only one subtype is common to all the studies, the subtype scoring low on all tests and evidencing severe motor impairment (global and fine). It is impossible to identify pure subtypes in small samples when the inclusion and exclusion criteria are unclear and not homogeneous (for example, specifying whether or not it is the first evaluation, what previous medical treatment or remediation was implemented, inclusion or exclusion of ADHD (Attention Deficit Hyperactivity Disorder) subjects, signs of pervasive development disorder, ASD (Autism Spectrum Disorder), prematurity, language delay... etc ). Only the study by Green et al.[9] and VaivreDouret et al. [11,20] screened with DSM for ADHD and ASD to control the presence of these co-morbidities or co-occurences and excluded these children from the first cluster analysis.

Furthermore, the assessment of cerebral functions among DCD children was frequently incomplete to understand if others relevant impairments in sensory-motor and cognitive functions could explain the gestural disturbance, and screening for neurological soft signs was rarely performed. When this screening was performed, the measures used in literature were the COMPS (Clinical Observation of Motor and Postural Skills [21] as in the study by Green et al.., [9], the Quick Neurological Screening Test [22] in the study by O'Hare \& Khalid, [23], or a non-normative assessment such as the PANESS examination [24-25]. However, no cluster study took the neurological soft signs into account in the DCD subtyping analysis, except for the studies by Vaivre-Douret et al.[11,12,19-20].

Because it is disappointing to read in 2019 the experts consensus, in the recent relevant document of international clinical practice recommendation (CPR) for developmental coordination disorders, initiated by the European Academy of childhood disability (EACD) who updated recommendations based on literature researches on assessment of DCD [26], that there is no analyse of DCD subtyping studies, and in addition a lack of knowledge or a negligence of neurodevelopmental French standardised assessments of body structure and functions. It has to be recognised that only two studies [11-12] are the first to use strict inclusion criteria and a standardized neurodeveloppemental battery of neuromotor and psychomotor tests (NP-Mot battery, with age-related normative data), [18, see table 1$]$, in addition with multidimensional standardized assessment of cognitive and neurovisual functions contributing to defining clusters with specific impairments for a better understanding of the nature of subtypes and co-morbidities/cooccurences in DCD. The NP-Mot developmental assessment makes it possible to take into account the maturation of gesture measured via normed qualitative (movements) and quantitative (speeds) assessments for each item and each function scored with identical independent subtests across ages. The NP-Mot battery [18] is a developmental standardized normative instrument which differs from the $M-A B C$, or BOTMP : by the fact there are identical subtests (qualitative et quantitative developmental norms) across ages and there is not a global score from the battery. The M-ABC [16] used in these cluster studies consisting of eight items grouped in three sections (manual dexterity, ball skills and balance) with a total impairment score. The BOTMP [15], consists of eight subtests, with a total of 46 tests items. Four of these subtests combine to form the gross motor composite score, and three subtests combine to form the fine motor composite score. These 
Vaivre-Douret L (2019) Specific impairments and predictive markers for developmental coordination disorder subtypes in children:The importance of multidimensional developmental assessments in cluster analysis

seven subtests, together with a subtest of upper limb coordination, form the battery composite score.

The developmental particularity of the NP-Mot (Table 1) is to consider the notation of quantitative and qualitative aspects of performance in the scoring, such as the placement of the arms when jumping, flying and postural control on landing (able to stop or not...). It also considers by example for static balance on one foot, qualitative score for participation of the upper limbs held away from the body, and quantitative score with timed standing on one foot. Thus, the score is dependent on age and related to the maturation of the central nervous system. It allows to detect mild or moderate to severe motor coordination impairments based on age, often never systematically observed and noted. On the contrary, performance tests such as M-ABC passes false negatives at younger ages and false positives at advanced

Table 1. Assessment battery of developmental neuro-psychomotor functions in children from 4 years old (NP-Mot, Vaivre-Douret, 2006) : components of the examination in bold. (Standardized functional neuro-psychomotor assessment with mean, deviation standard or standard score for each subtest with qualitative (movement) and quantitative (timing) maturation score according to age)

Neuromuscular tone examination/ neurological soft signs: $R / L$ developmental scores

-Passive muscular tone:

1.Resistance and degrees angle measures for passive extensibility of shoulders, wrist, trunk, adductor angles, heel-ear angle, popliteal angle, dorsiflexion angle of foot (detection of hypotonia/ hypertonia, abnormal phasic stretch reflex in one or both gastrocnemius muscles/motor pathway

2.Resistance of slack or dangling hands and feet

3. Knee jerk reflex

-Active tone / dysdiadochokinesis and synkinetic movements (co-movements and mirror movements):

1. Rapid pronation and supination of the hand

2.Repeated opening and closing of both hands

3. Repeated opening and closing the mouth.

-Standing/Sitting tone: Pushes

Laterality

1. Tonic laterality of upper and lower limbs (homogeneous dominance) (see tone examination/resistance corresponding to dominance side of hand and foot)

2. 4 Spontaneous gestural laterality of upper limbs (R/L) (e.g. place one fist over the other)

3. 10 Psychosocial laterality of hand preference (representation of transitive/intransitive gestures on verbal command)

4. 3 Usual laterality of upper limbs (e.g. Rub out a cross in the middle of a page) and for lower limbs 4 kicks in a ball

5. Dominant director eye (task with a cone)

Gross motor control: Timing and quality scores

1. Static balance (feet together /on one foot)

2. Dynamic balance (spontaneous walk, walk on a line heel -toe and back, tiptoe, heels, jump from a podium feet together)

(+score for global coordination between limbs and/or for postural control on jump landing)

Praxis (repetitive and alternating movements): Timing and quality scores

1. Bimanual coordination: pronation-supination, with symmetrical and asymmetrical movements

2.Digital praxis:

. Index finger-thumb (20 movements)

. Successive touching thumb to fingertips for each hand (20 movements)

3. Gnoso-praxis- imitation of gestures (Vaivre-Douret, 2002): Imitation of 10 hand gestures and 16 finger gestures (Fig 1)

4. 10 Representational gestures (cf Psychosocial laterality): quality (primitive/ symbolic) of the gesture.

5. 7 Oro-facial praxis

6. 5 questions about dressing

Digital perception

Localization of digital tactile stimuli (right/left hand)

Manual dexterity

Putting the row of twelve counters one by one into a box, for each hand: score of timing and quality

Bodily spatial integration (right from left)

- - In relation to self (4 items)

- Verbal command with axial crossing gesture (4 items)

- Pointing on the examiner ( 2 items)

- Pointing on a doll (2 items)

- Imitation of examiner with axial crossing gestures ( 8 items)

- Objects (8 items with two and three objects)

- Map directions (turn right/left)

Rhythmic tasks

- Spontaneous rate of regular hand taps; On 21 taps /timing and gestual regularity

- Auditory-visual-kinaesthetic adaptation task via imitation of hand/foot tapping patterns

- Auditory-perceptual-motor rhythmic adaptation (clap, walk): with metronome speed at 90, 60 and 120

Auditory-attentional task

Taps in a "go/no-go" task (1 tap/ 2 taps): Series of 16 taping with a chopsticks (timing and quality scores) 
Vaivre-Douret L (2019) Specific impairments and predictive markers for developmental coordination disorder subtypes in children:The importance of multidimensional developmental assessments in cluster analysis

Neuromuscular tone examination/ neurological soft signs: R/L developmental scores

-Passive muscular tone:

Resistance and degrees angle measures for passive extensibility of shoulders, wrist, trunk, adductor angles, heel-ear angle, popliteal angle, dorsiflexion angle of foot (detection of hypotonia/ hypertonia, abnormal phasic stretch reflex in one or both gastrocnemius muscles/motor pathway

Resistance of slack or dangling hands and feet

Knee jerk reflex

-Active tone / dysdiadochokinesis and synkinetic movements (co-movements and mirror movements):

1. Rapid pronation and supination of the hand

2.Repeated opening and closing of both hands

3. Repeated opening and closing the mouth.

-Standing/Sitting tone: Pushes

Laterality

1. Tonic laterality of upper and lower limbs (homogeneous dominance) (see tone examination/resistance corresponding to dominance side of hand and foot)

2. 4 Spontaneous gestural laterality of upper limbs (R/L) (e.g. place one fist over the other)

3. 10 Psychosocial laterality of hand preference (representation of transitive/intransitive gestures on verbal command)

4. 3 Usual laterality of upper limbs (e.g. Rub out a cross in the middle of a page) and for lower limbs 4 kicks in a ball

5. Dominant director eye (task with a cone)

Gross motor control: Timing and quality scores

1. Static balance (feet together /on one foot)

2. Dynamic balance (spontaneous walk, walk on a line heel -toe and back, tiptoe, heels, jump from a podium feet together)

(+score for global coordination between limbs and/or for postural control on jump landing)

Praxis (repetitive and alternating movements): Timing and quality scores

1. Bimanual coordination: pronation-supination, with symmetrical and asymmetrical movements

2.Digital praxis:

. Index finger-thumb (20 movements)

Successive touching thumb to fingertips for each hand (20 movements)

3. Gnoso-praxis- imitation of gestures (Vaivre-Douret, 2002): Imitation of 10 hand gestures and 16 finger gestures (Fig 1)

4. 10 Representational gestures (cf Psychosocial laterality): quality (primitive/ symbolic) of the gesture.

5. 7 Oro-facial praxis

6. 5 questions about dressing

\section{Digital perception}

Localization of digital tactile stimuli (right/left hand)

Manual dexterity

Putting the row of twelve counters one by one into a box, for each hand: score of timing and quality

Bodily spatial integration (right from left)

- $\quad$-In relation to self (4 items)

- Verbal command with axial crossing gesture (4 items)

- Pointing on the examiner ( 2 items)

- Pointing on a doll (2 items)

- Imitation of examiner with axial crossing gestures ( 8 items)

- Objects ( 8 items with two and three objects)

- Map directions (turn right/left)

Rhythmic tasks

- Spontaneous rate of regular hand taps; On 21 taps /timing and gestual regularity

- Auditory-visual-kinaesthetic adaptation task via imitation of hand/foot tapping patterns

- Auditory-perceptual-motor rhythmic adaptation (clap, walk): with metronome speed at 90, 60 and 120

Auditory-attentional task

Taps in a "go/no-go" task (1 tap/ 2 taps): Series of 16 taping with a chopsticks (timing and quality scores)

ages [27]. Furthermore the NP-Mot battery includes standardized examinations of neurological soft signs (neuromuscular tone and neuromotor examination), in line with earlier studies of minor brain dysfunction and predictors of learning or movement disorders [28]. Thus, the NP-Mot aims to capture maturational aspects in the ability to perform and quality of performance, reflecting the development of neurological mechanisms contributing to movement timing, motor control, motor coordination, and motor execution. The M-ABC targets general motor performance with items involving dual tasks with training that are quite difficult to perform, requiring different cognitive functions to succeed (memory, attentional, and emotional functions).
This means that the M-ABC does not provide an understanding of the motor deficit because the measure does not provide information making it possible to identify the difficulties affecting the motor performance. Thus, the M-ABC requires caution, since it can lead on the one hand to false negatives and on the other to failure to identify minor motor deficits (criterion D of DSM-5) that can affect academic and daily living performances at home and at school. Complementary neuropsychological cognitive tests and neurovisual may be included to investigate learning difficulties (executive, memory, attention, visual perception) commonly associated with DCD $[11,12,14,19]$. 
Vaivre-Douret L (2019) Specific impairments and predictive markers for developmental coordination disorder subtypes in children: The importance of multidimensional developmental assessments in cluster analysis

The choice of appropriate measures in methodology of the study and informations about characteristics of included participants have an impact on understanding of the nature and etiology of different subgroups of DCD $[11,20]$.

\section{Contribution of multidimensional assessments in a cluster study (Part I) and multivariate analysis with supervised statistical models (Part II)}

We report here an overview of contribution of multidimensional assessments in cluster studies on DCD subtypes $[11,19]$ in order to a better understanding of the methodology used.

Two (non-independent) samples of DCD children were used in these analyses: an initial sample of 43 children (mean age $8.3+2.3$ years) (Study Part I) [11], and a sample augmented with 20 children of similar ages (Study Part II) [12], giving a total of 63 children.

A total of 63 DCD children (52 (83\%) males and 11 (17\%) females) aged between 5 and 15 years, median age 8.1 ( 8.5 for males and 7.8 for females) were enrolled in a prospective study previously described $[11,12]$. Diagnosis was based on DSM-IV-R criteria (criterion A, B, C and D) which are essentially the same in the current DSM-5 [1]. All children met DSM-5 criteria.

The children were screened during a first out-patient clinic consultation in a Paediatric and in a Child Psychiatry department in Cochin and Necker Hospital AP-HP, Paris, France.

The children's parents answered a questionnaire on medical and behavioural characteristics. The children recruited for the study had undergone no previous assessment, medication, or therapy, and had no known medical abnormalities no physical, neurological or sensory deficits known, no current diagnosis of attention deficit hyperactivity disorder (ADHD), and no Autism spectrum disorder based on DSM-5 criteria. Any child born premature ( $<37$ weeks). IQ levels were in the normal range (the median Full IQ, Verbal IQ and Performance IQ were 100,110 and 90 , respectively).

\section{Measurement of study variables}

Neuropsychological assessment: The neuropsychological assessment consisted of subtests from the standard Wechsler measure of intelligence, and standardized tests (previously described in VaivreDouret et al.., [11]) to assess visual-constructional skills [29], visualspatial structuring (copying Rey's complex geometric figures, and Beery's Visual-Motor Integration test [17] consisting in copying 2D geometric graphic representations), visual-spatial attention (bell-crossing test and $\mathrm{O}$ linear crossing standardized test), and mental planning executive functions (Porteus Labyrinth and Tower of London test, [30]). Impaired handwriting was characterized by spatial disorientation (alignment, letters irregular in shape) and sentences that were not organized on the page of the school exercise book, with spacing problems. We correlated these observations on poor handwriting with specific tasks of visualmanual reproduction of cycloids, epicycloids and hybrid loops, exploring the maturation of the organisation of handwriting gestures (upper limb, segmental organization of fingers, hand, forearm, arm, and shoulder), [31,32]. In these children, handwriting is characteristically poor, and they have more difficulty in copying. The de Ajuriaguerra et al.handwriting scale [33] was also used to detect dysgraphia among the children. It is similar and correlated (0.78) to the Concise Evaluation Scale for children's handwriting [34]. Visual perception was assessed using form recognition tasks [35], tangled lines and stylised outlines of animals, assessing visual oculomotricity perception and visual gnosis respectively (100\% success at 4 years old). Kinaesthetic perception was assessed with a status memory test assessed by positioning the child's arm and finger and asking him with eyes closed to remember and repeat. In addition, two French language batteries [36] and the N-EEL battery by Chevrie-Muller \& Plaza [37] were used, including tasks of reading, repetition of words and logatoms, picture-naming speed, meta-phonological tests, auditory memory and working memory tasks (digit span).

Neuropsychomotor developemental assessment: The neuropsychomotor developemental assessment was based with agerelated normative data from the assessment of the NeuroPsychomotor functions in children battery (NP-Mot) from 4 years old, [18], which measures developmental maturation from the following: neuromuscular tone examination and soft neurological signs, gross motor-control tasks, laterality, bimanual and digital praxis, digital perception (digital gnosis), manual dexterity, bodily spatial integration, rhythmic tasks, and auditory-attentional tasks (Table 1). Scores for each subtest and function investigated are expressed as standard deviation and standard score. Overall test-retest reliability of the NP-MOT has been reported to range from 70 to $98 \%$, described in the test manual [18], and correlation coefficients with the Bruininks-Oseretsky Test of Motor Proficiency [15] for upper-limb coordination, balance, and bilateral coordination subtests were 0.72 and 0.84 . In addition, like Ayres [38] we administered a standardized (developmental norm-referenced) imitation of gestures test [39], involving non-meaningful hand $(n=10)$ and finger positions $(n=16)$ to be copied from the examiner. The posture to be imitated is sustained by the examiner until the child has succeeded in reproducing the gesture. The specific items for hands and fingers are shown in Figure 1. The score takes into account the recognisable reproduction of the spatial posture and incorporates the qualitative factor of movement planning [39]. Thus, when the child reproduced the model in a single step, the score was 1 and when it was via a step-by-step construction the score was 0.5 . In case of inability to execute the required model, the score was 0 . When the child was not able to imitate the gesture with his own hand, we asked to the child to reproduce the gesture on the examiner's hand. The posture to be reproduced in this case is sustained by another examiner.

Other measures: Finally, an electro-physiological visual examination including electro-retinogram (ERG), visually evoked potentials (VEP) and motor electro-oculogram (vertical and horizontal pursuit) was performed in order to study the sensory and visual motor pathways $[18,40]$.

Procedure: Participants provided written informed consent before the start of the study. The studies were approved by the Institutional Review Board of the University Ethics Committee.

The children recruited were born full term ( $>37$ weeks), with onset of clumsiness or motor symptoms in the early developmental period as reported by the parents (difficulties in motor acquisitions, or/and in fine motricity with block-building or constructive manipulatory play, such as Lego blocks following a model or/and completion of puzzles, difficulties in dressing). Thus, all the children demonstrated moderate to marked impairment in early development of motor skill coordination and/or motor coordination (e.g. failure $<1$ SD in DFMOT standardized motor skills, global and fine, [41] or in NP-MOT assessment of global coordination (DSM criterion A). All the children showed manual praxis deficits [18], and/or poor handwriting on the handwriting scale [33] and/or in visuo-motor integration tasks (VMI), [17] without visual perceptual difficulties on the Frostig standardized test [35], interfering with the performance of daily activities and with academic achievement (DSM criterion B). 


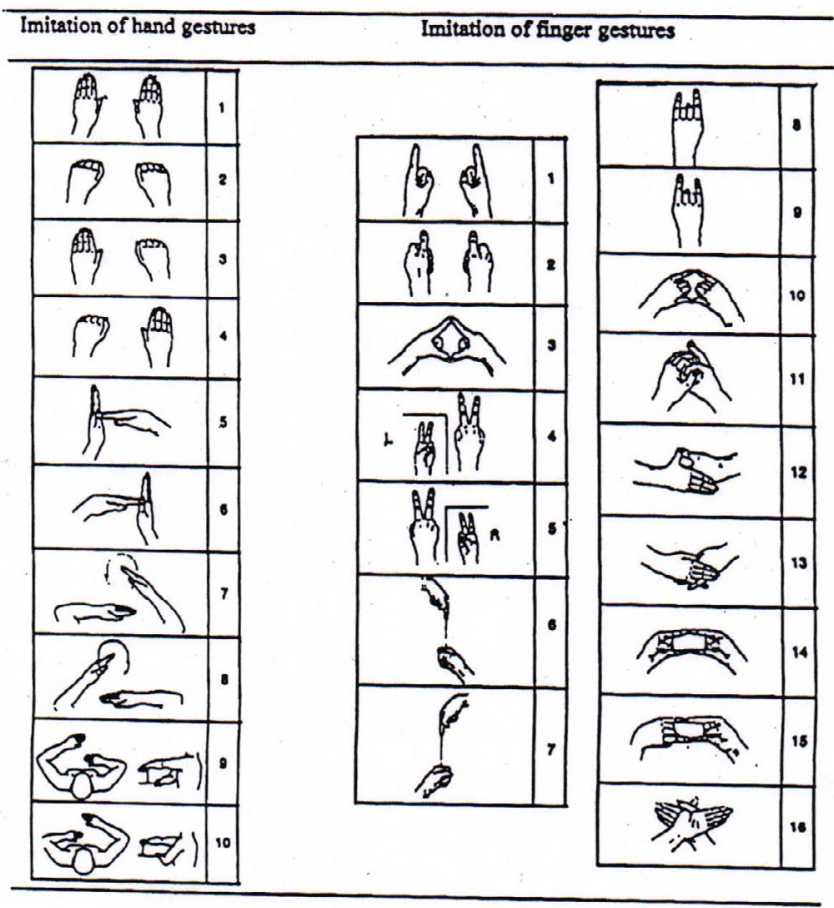

Figure 1. Items for hands (10) and fingers (16) in the gesture imitation test [39] (All items are a view from the examiner, Items for hands $\mathrm{n}^{\circ} 9$ and 10 are executed by the examiner while the child closed his eyes and open them to do the imitation)

First, the questionnaire from Geuze's research on academic performance and activities of daily living (criterion B) was administered to all the children [42]. Furthermore, the parents of the children with DCD were asked a few questions about pregnancy, age at the time of the first motor milestones (i.e. sitting alone, crawling, walking alone, first sentences, noted in the health booklet), about the child behaviour and social competences, medical and family history, difficulties in leisures and play with constructional manipulatory play, such as puzzles and Lego blocks, and academic performances (arithmetic, reading) noted in the school reports. In the second phase, extensive neuro-psychological and neuro-psychomotor evaluations followed by neuro-visual examination were administered. All test scores were standardized following the authors' scoring guidelines. They all are expressed as either deviation from population mean in standard deviation units (failure if $<1 \mathrm{SD}$ ) or as a score distribution (failure if $<$ age-adjusted 20th percentile) depending on the test under consideration. Thus, all the children recruited for this study met DSM- 5 criteria A, B and C, and completed all subtests for the different investigations.

\section{Statistical analyses}

All statistical analyses were carried out using the $\mathrm{R}$ statistical package [43].

The same binary (not impaired/impaired) measures were collected in both samples of studies $[11,12,19]$. The statistical analyses were not adjusted for age (low variance, 2.5 years) nor for gender given the strong imbalance between males (83\%) and females (17\%) for a small sample size.

In Studies Part I [11], an unsupervised approach was chosen. Hierarchical Ward-based clustering and the k-means algorithms were used to identify 3 groups of subjects sharing similarities in terms of impairments on a set of 49 binary measures. The number of clusters was selected according to various validity criteria (Dunn's index, cophenetic correlation, Hubert Gamma index and the Gap statistic $[44,45]$. Hierarchical clustering is well-suited to handling samples of moderate size $(\mathrm{n}<100)$. Basically, this bottom-up strategy starts at the bottom (each individual) and at each level recursively merges a selected pair of clusters into a single cluster. The resulting cluster consists in the two groups of subjects with the smallest inter-group dissimilarity. Thus, cluster results rely on the choice of an appropriate distance measure. Furthermore, deciding on the number of clusters or the way to assign subjects to clusters mostly relies on graphical interpretations of the cluster results or validity indices. Visual-guided criteria include the analysis of the dendrogram, which is a visual picture of the aforementioned agglomerative steps: each 'leaf' represents a given individual, and the individuals that are the most alike (according to the chosen distance) are joined together on what is called a node. Thus, selecting a given number of nodes amounts to selecting a corresponding number of clusters. It can however be noted that a dendrogram is a tree structure; the cophenetic correlation coefficient can be used to assess the degree to which the dendrogram preserves the pairwise distance between the original data points. Numerical criteria, also known as validity indices, can be used instead. These are data-driven criteria that have been shown to be effective cluster validity estimators in various clustering applications. Hubert's Gamma is a measure of the correlation between the chosen distance and a binary vector where 0 means that individuals belong to the same cluster and 1 that they are from different clusters. This index is computed after cutting the tree at each node, and retaining the number of clusters for which Hubert's Gamma is maximal. The Dunn index [46] defines the ratio between the minimum intra-cluster distance and the maximum inter-cluster distance and it should also be maximized. Iterative methods such as the k-means are more efficient for small to moderate sample sizes or as a pre-processing step before applying hierarchical clustering. Here, $\mathrm{k}$-means are used to find clusters in the data in a non-deterministic way. As the number of clusters, or equivalently the center coordinates, must be specified during the initialisation of this algorithm, one generally tests an increasing number of clusters, checking how the residual sum of squares (i.e. the variance unexplained by the cluster structure) evolves. Another way to circumvent the arbitrariness of starting values consists in running several trials (typically 20 to 30 ) on the k-means and choosing the solution that minimizes intra-class inertia for a given k-cluster solution. As an alternative to the minimization of the residual sum of squares or intra-class inertia, one can also use the Gap statistic [45] which reflects change in within-cluster dispersion with that expected under a reference null distribution. The stability of the resulting cluster solutions was assessed using bootstrap resampling as implemented in the fpc R package [47].

In Study Part II [12,19], a supervised approach was retained, based on the clinical subgroups delineated in Study Part I. In order to select the most informative markers on the whole sample $(\mathrm{N}=63)$, a feature selection was performed based on Random Forest ${ }^{\mathrm{ma}}$ (RF) and Partial Least-Squares discriminant analyses (PLS-DA) with and without L1norm penalty (lasso). A nested 10x5 cross-validation design was used (0.7:0.3 split ratio for the training and validation subsamples) to tune the hyper-parameters of those two models (number of variables used to build trees with RF, L1 penalty for PLS-DA), with average classification accuracy as the main criterion on the training sample. Measures of variable weight were computed for each model (mean decrease in accuracy for RF, variable loadings for PLS-DA), and statistical significance was assessed using 1000 permutations. 
Vaivre-Douret L (2019) Specific impairments and predictive markers for developmental coordination disorder subtypes in children:The importance of multidimensional developmental assessments in cluster analysis

\section{Results}

Overall, there were 50 items, scored as successful/failed for each participant. The inspection of marginal distributions showed no particular asymmetry, except for one item (neurovisual ERG) which was always scored as successful. As this item lacks discriminant power and provides no information about patients it was discarded from further analysis, leaving a total of 49 items.

\section{Study Part I [11]}

The results from cluster analyses (Ward-based hierarchical and $\mathrm{k}$-means clustering) suggested a three-cluster solution (respectively $\mathrm{n}$ $=5,21$ and 17 subjects, figure 2), which was found to be acceptable according to several validity indices (cophenetic correlation $=0.549$, Hubert Gamma $=0.545$, Dunn index $=0.638$ ). Ward's minimal distance (variance) criterion and the squared Euclidean distance provided monotonous distance measures, which is desirable in this situation. They were used, for instance, by Hoare [5] and Macnab, Miller \& Polatajko [8], since they also facilitate comparisons with the k-means procedure which is also based on squared Euclidean distances.

However, to assess the reliability of the squared Euclidean distance measures, these results were compared to those obtained using the simple Euclidean distance or the Jaccard distance which is more appropriate to binary response vectors. The use of different distance measures (Euclidean, squared Euclidean, Jaccard for binary variables) yielded comparable results, and the three-class solution was also clearly identified using $\mathrm{k}$-means. In this case, there was no improvement in the Gap statistic when more than 3 classes were envisaged, and the threecluster solution exhibited lower intra-class variance. Overall, only 4 children were misclassified, among whom there were no children with ideomotor impairment (Figure 2).

Bootstrap resampling (500 samples) was used to assess the variability of the cophenetic correlation for the original Ward-based hierarchical clustering, and it was found that inter-individual distances were well conserved in the clustering procedure (cophenetic correlation $=0.638,95 \% \mathrm{CI},[0.545 ; 0.725])$.

It is worth noting that Ward-based hierarchical clustering on the whole sample yielded comparable results to those discussed in VaivreDouret et al. [11].

The mean profiles of impairment for each subgroup are summarized in Figure 3. Impairment is based on scores at least one standard deviation below the mean or on age-adjusted percentile $\left(<20^{\text {th }}\right)$ for some cognitive tests in accordance with standardized instruction and developmental norms. These cluster profiles evidence two "pure" DCD subtypes : $8 \%(n=5)$, relating to Ideomotor disorders (IM), 52\% $(n=33)$ relating to visuo-spatial and constructional motor impairment (VSC), and the remaining $40 \%(n=25)$ formed a mixed group sharing impairments common to IM and VSC, and with additional co-morbidities or co-occurence.

\section{Study Part II $[12,19]$}

The results of feature selection were identical between Random Forest (RF) and sparse PLS-DA. In the case of RF, variable ranking based on re-randomized measures of variable weight (see Lalanne et al.., [12] for details) suggested eight significant items ( $\mathrm{p}<0.05)$ : digital

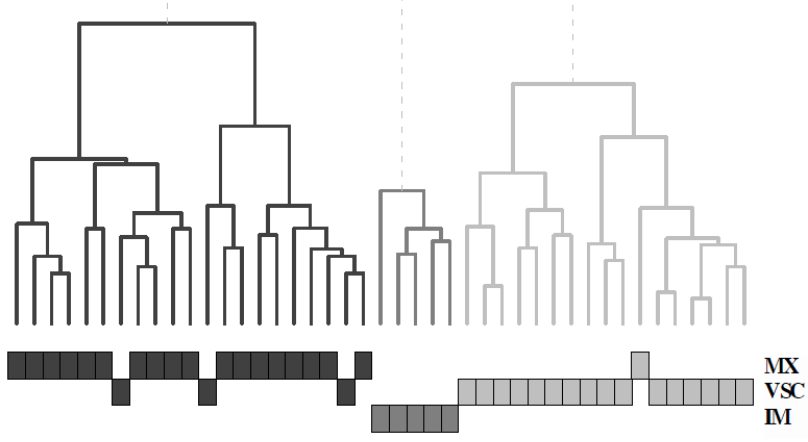

Figure 2. Dendrogram resulting from Ward hierarchical clustering. Clinical subgroups (MX, mix DCD; VSC, visuo spatial and constructional and IM, ideomotor DCD children) are indicated in the lower panel to help visualize the misclassification rate

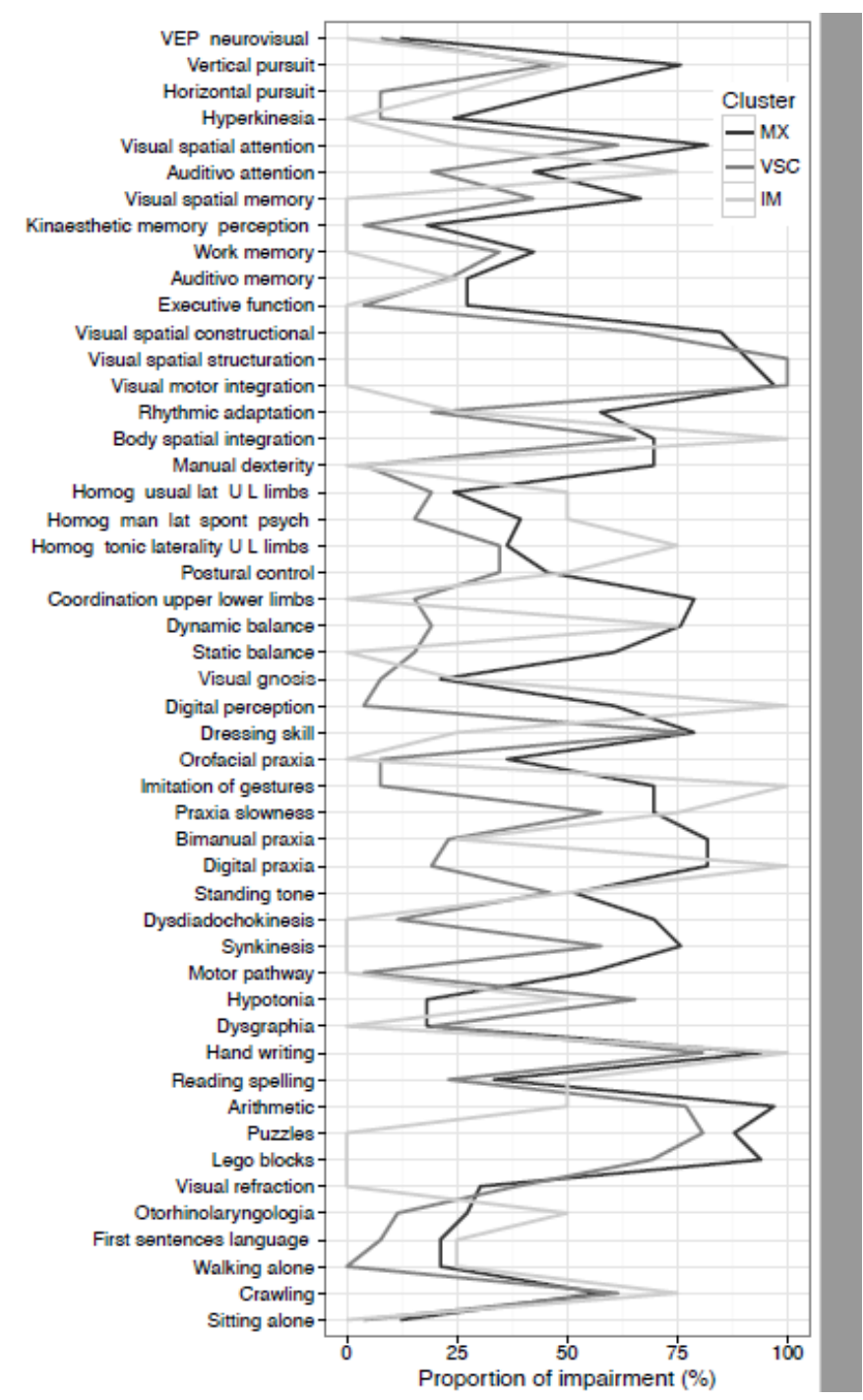

Figure 3. Items scores (\% Failure) for cluster-based subtyping of DCD. (Mean profiles for each subgroup (IM, ideomotor; VSC, visuo spatial and constructional; MX, mix DCD) defined by impairment on a 49 -items assessment battery. Items from "sitting alone" to "arithmetic" belong to the questionnaire issued to parents) 
Vaivre-Douret L (2019) Specific impairments and predictive markers for developmental coordination disorder subtypes in children: The importance of multidimensional developmental assessments in cluster analysis

praxis, imitation of gestures, digital perception, visual motor integration and Lego blocks, visual spatial structuring, manual dexterity, and coordination between upper and lower limbs. Details about the way the statistical significance of these items was assessed are available in Lalanne et al.[12]. Classification accuracy on the validation sample was perfect in the case of RF (100\%) and very good for PLS and sparse PLSDA (91.4\%, [71.3-99.9]).

The inspection of conditional and marginal distributions of impairment variables yields three main characteristic classes of impairments. IM children were equally impaired on digital perception, imitation of gestures and digital praxis. Some impairments were shared across VSC and MX children - Lego blocks, visual spatial structuration, visual motor integration. Some impairments were specific to MX children - digital praxis, digital perception, imitation of gestures, coordination between upper and lower limbs.

\section{Discussion}

\section{Analysis of methodological aspects of all the cluster studies in literature}

In all nine cluster studies in the literature, sets of measures were acquired and subjects were grouped together on the basis of the profiles of their scores $[2,4-9,11,14]$. However, it can be noted that the subtypes reported by these studies differ as to the number and the characteristics of the subjects assessed, and the assessment measures used and the cut-off. These methodological choices can be important for the interpretation of the cluster structures obtained (for a review, see Macnab, Miller, \& Polatajko) [8].

First, the inclusion criteria in these studies are often not clear for different reasons. In the DSM criteria, no cut-off criterion is specified. Thus, for example, studies using the $\mathrm{M}-\mathrm{ABC}$ test [16] considered a range in impairment scores from the 5 th to the 15 th percentiles, involving severe to borderline impairments, based on total performance scores corresponding to different profiles of performance (gross motor versus fine motricity) [48]. Furthermore, the inclusion of children in the different studies drew on different populations, which could introduce a bias in cluster analysis (e.g. learning disabilities, selection by teachers, inclusion in motor remediation program...etc), and only some studies considered co-morbidities [8]. Nor do these studies always provide information on any medication or remediation.

Concerning the number of clusters found in the different studies, there was considerable variability, with respectively 6 clusters for Lyytinen \& Ahonen [2]; 4 for the studies by Miyahara [6], Dewey \& Kaplan [4] and Wright \& Sugden [7]; 5 for Hoare [5], Macnab et al.[8] and Green et al.[9]; and 3 main clusters for Vaivre-Douret et al.[11]. The reason for this variability is the choice of measures, for example only the motor subtests from the BOTMP [15] were used in the Miyahara study [6], or M-ABC subtests for Wright \& Sugden [7], while Hoare [5] and Macnab et al.[8] included in addition other variables such as kinaesthetic acuity, visual perception, and visual motor integration. Furthermore, , cluster scores were standardized in relation to sample mean and standard deviation (the zero line representing the mean for the entire sample) as in Hoare [5] or Macnab et al.[8] to the population norms as in the Miyahara study [6], or to age adjusted on developmental norms as in Vaivre-Douret \& al. [11].

Thus, no homogeneous subtype running across all these studies can be systematically compared, which explains the difficulty in understanding the nature of the different profiles of children with DCD.
In all studies, there is however one group with poor balance and another with difficulties in all areas of perceptual-motor skills (including fine, gross motor, visual-spatial skills) [4 6,9]. Also, the severity of the motor performance deficits was associated with the degree of the visual-motor impairment in the clusters in the studies by Hoare [5] and Macnab et al.[8]

\section{Specific impairments with multidimensional assessments}

The findings of the cluster studies [11-12] aim at constructing a complete semiology with specific markers for DCD children based on extensive standardized assessments - neuromotor, neuro-psychomotor, neuropsychological and neurovisual. This was intended to highlight distinct profiles on a total of 49 assessment items scored as passed/ failed. The study first [11] of all enabled the identification of three main significant subtypes, comprising two "pure" groups, Ideomotor, (IM) and Visual-Spatial/Constructional (VSC), and a "mix" group sharing impairments with the first two groups and including additional comorbidities (MX). Secondly, the Part II study [12] identified predictive criteria for these DCD subtypes by augmenting the sample. The focus here was on isolating specific markers with high predictive discriminant power $[12,19]$.

These results contribute in significant manner to the current debate on the semiology of DCD subtype profiles and enable a possible interpretation of the mechanisms involved.

The research by Vaivre-Douret \& al. [11-19] and Lalanne et al.[12] conducted in-depth analysis of 49 developmental items, reported in figure 3 (motor and language development, motor manipulatory play and academic abilities (spelling, reading, handwriting and arithmetic), neuropsychomotor, neuropsychological/ neurocognitive investigations, neurovisual examination). This made it possible to establish the distribution of impairments in the different profiles specific to two "pure" subtypes (IM and VSC) versus the "mixed" subtype MX from the cluster analysis in figure 3.

It can therefore be said that most of the studies in the literature have not made it possible to identify the specifically impaired characteristics and have only identified subtype profiles showing poor performances relative to the overall sample. These are rarely more than one standard deviation below the mean, except for one cluster common to all the studies with generally low scores on all the items. But none of these studies in literature do not jointly investigate praxis, visual perception, visuomotor integration and global motricity in relation to developmental qualitative and quantitative factors (norm-referenced), so this common cluster embraces motor coordination disorders as a whole without providing any understanding of the nature of the motor impairments involved. Moreover, the motor evaluation is often based on the M-ABC [16] and on total performance scores evened-out between gross and fine motor skills, without differentiating the nature of the disorders (quality/timing). Some children may not to be recorded as presenting motor impairments, or there may be false negatives relative to the difficulty of items. Thus, the M-ABC appears to have limited relevance in diagnosis of children with DCD.

The most discriminant markers (significant, $\mathrm{p}<0.05$ ) to explain the specificity of the three subtypes evidenced in part II of the study [12] are: digital praxis, imitation of gestures, digital perception, visual motor integration, manual dexterity, visual spatial structuration, coordination between upper and lower limbs, and Lego blocks. This was confirmed by two multivariate statistical models (100\% with Random Forest (RF) and 91,4\%, (0.713-0.999) with PLS discriminant analysis) [12,19]. 
Vaivre-Douret L (2019) Specific impairments and predictive markers for developmental coordination disorder subtypes in children: The importance of multidimensional developmental assessments in cluster analysis

These results suggest that it is possible to consider a single subset of items from the 49 assessed, while at the same time preserving acceptable diagnostic quality. They also suggest that the children in each diagnostic class formed in this manner correspond to wellcharacterized, homogenous groups. Thus, IM children are significantly more likely to fail on tests such as digital perception, imitation of hand and finger gestures [37, Figure 1] and digital praxis (Table 1). Certain markers are common to the VSC and MX groups, in particular visualspatial structuring, Lego blocks, puzzles, visual-spatial constructional and visual-motor integration skills, arithmetic, and dressing skills, while other markers such as manual dexterity, dysdiadochokinesis, and coordination between upper and lower limbs are specific to the MX category (Table 2).

\section{Identification of three main subtypes of DCD with multidimensional assessments $[11,12,19]$ and neurophysiological brain mechanisms involved}

The ideomotor impairment subgroup (IM): In the IM group $[11,12,19]$, there are significant impairments compared to the VSC group $(\mathrm{p}<0.05)$ (Figure 3 and Table 1$)$ concerning digital praxis, gnosopraxis (imitation of hand and finger gestures) and digital perception. A certain slowness in praxis is observed, along with impaired dynamic coordination, mainly for postural control. It can be noted that in the imitation of gestures (finger movements), less well-performed, the subjects were able to imitate the model on the examiner's hand. This shows that the visual perception of another person's hand is unaffected. Further to this, it is in this group that significantly more frequent ear, nose and throat disorders were observed than in the VSC group, more frequent language delays and more frequent difficulties in reading. This recalls Nicolson [49] and O' Hare \& Khalid [23] who have underlined a high risk of reading delay in DCD. Global or axial hypotonia, disorders in spontaneous gestures and the tonic organisation of laterality, and poor left-right integration of bodily spatial orientation (especially in imitation in relation to another person), were all variables that were prominent in this group, reflecting difficulties in the bodily integration of the egocentric axial reference [50]. This is linked to axial hypotonia, frequently observed in our study but never in the literature, because the examination of muscular tone is never assessed. When arithmetical skills (academic achievement) were affected in this group, it mainly concerned the acquisition of multiplication tables, and thus the sequential aspect. The identification of this group is in line with other research [4,39,51-53], evidencing difficulties in non-habitual or sequential movements. These difficulties in the IM group, from a semiological viewpoint [13], suggest motor planning difficulties partly

Table 2. Constitutiion of three main subtypes based on the interpretation of failure to specific items [11-12,19]. (* Verbal IQ always $>$ Performance IQ, $\mathrm{p}<.001$

\begin{tabular}{|c|c|c|c|}
\hline \multicolumn{2}{|c|}{$\begin{array}{c}\text { Ideomotor } \\
\text { (IM) }\end{array}$} & $\begin{array}{c}\text { Visual spatial / } \\
\text { constructional } \\
\text { (VSC) }\end{array}$ & $\begin{array}{c}\text { Mix } \\
\text { (MX }=\text { (IM + VSC + co- } \\
\text { morbidities) }\end{array}$ \\
\hline N & $\mathbf{5}$ & $\mathbf{3 3}$ & $\mathbf{2 5}$ \\
\hline Age & $8.2 \pm 2.6$ & $7.3 \pm 1.5$ & $9.1 \pm 2.7$ \\
\hline Male & $2(40 \%)$ & $19(90 \%)$ & $14(82 \%)$ \\
\hline Full IQ* & $109.0 \pm 4.9$ & $83.0 \pm 21.2$ & $111.9 \pm 19.7$ \\
\hline Specifics & $\begin{array}{c}\text { Imitation of } \\
\text { gestures** }\end{array}$ & Visual motor integration** & Manual dexterity $\dagger$ \\
\hline Items & $\begin{array}{c}\text { Digital } \\
\text { praxia** }\end{array}$ & Visual spatial structuring** & Coordination between \\
\hline Failure & $\begin{array}{c}\text { Digital } \\
\text { perception** }\end{array}$ & Lego blocks** & upper and lower limbs $\dagger$ \\
\hline
\end{tabular}

** Items differing between IM and VSC ( $p<.05$, controlled by FDR), $\uparrow$ Abnormalities specific of MX-DCD, (MX vs. IM+VSC, p<.05) linked somato-sensory difficulties and abnormalities in the regulation of tone and motor control in motor planning tasks, which implicate sub-cortical structures, including the thalamus, the basal ganglia and the cerebellum, as reported by Lundy-Ekman et al., [3]. This is in line with abnormal cerebellar function in DCD reported by O'Hare and Khalid [23] and the role of the putamen in the basal ganglia during a motor sequencing tasks (finger-thumb opposition task) reported in the positron emission tomography study by Seitz et al.., [54].

Visual-spatial and constructional impairment subgroup (VSC): A second "pure" DCD subtype group was identified $[11,12,19]$ : visualspatial and constructional DCD (VSC) (figure 3), showing significant, specific impairments $(\mathrm{p}<0.05)$ compared to the IM group (Table 2$)$, in the area of visual-spatial motor abilities - impairments in visual-motor integration, visual-spatial motor structuration, and performances on Lego blocks. Arithmetical skills were particularly affected in this group, for visual-spatial motor orientation (setting out a sum, constructing and completing a grid), and for visual-spatial motor organisation (positioning on a page, geometry). There were also the dressing abilities, mainly involving difficulties telling back from front, or right from left.

This visual-spatial/constructional DCD subtype has been evidenced in a few other cluster studies [5,8,9], using similar visualmotor perception tests. It can be noted that in this VSC group there are no significant motor coordination difficulties, but there are visualmotor difficulties. In addition, in this group, a larger proportion of visual refraction impairments was observed (myopia, astigmatism, hypermetropia) (55\%), which could have an impact on visual attention, but this does not explain VSC impairments, as also underlined by MonWilliam et al.[55]. Furthermore, the Creavin et al.study [56] showed in severe DCD that children have more co-occurring ophtalmological abnormalities.

Dysgraphia is noted in this group, possibly linked to visualperceptive motor disorders and ocular motricity (poursuits of eye movements), since in the VSC group we observed only $10 \%$ visual gnosis (perception) impairment and 5\% kinaesthetic perception impairment. Furthermore, dysgraphia is often associated with minor neurological dysfunction [32]. This is in line with the results obtained by Lyytinen \& Ahonen [2], Macnab et al. [8] and Green et al. [9], who showed a possible distinction between perceptive and motor functions in their cluster results. Our results are also in line with those of the study by Dewey \& Kaplan [4], Shoemaker et al. [57] and Van Waelvelde et al. [58], in favour of visual-perceptual motor difficulties. Thus, in the VSC group the difficulties appear to be specifically motor-perceptual, in association with established eye-pursuit disorder [40] and non-visual perceptual or sensory perceptual neurovisual disorders. As underlined by Milner \& Goodale [59] two sight systems should be distinguished, one oriented towards perception and identification (occipital-temporal ventral visual pathway for the question "What is it") and the other towards localisation and action (occipital-parietal dorsal pathway for the question "where"), responsible to lead with the vision a movement (perceptual-motor coupling). So, this second pathway is particularly impaired in the VSC subtype, involving the parieto-occipital lobe via parietal subregion of the corpus callosum, the cerebellum, the basal ganglia and thalamus, and the brainstem for the performance of eye movements. Obviously, a visual-perceptual deficit has an effect on gestures and can easily be confused with DCD, although the underlying brain mechanisms are not the same.

As VEP and ERG tests were virtually normal in this group, this confirms the absence of neurovisual perceptual impairments that might have explained the difficulties observed. In addition, explorations of 
Vaivre-Douret L (2019) Specific impairments and predictive markers for developmental coordination disorder subtypes in children: The importance of multidimensional developmental assessments in cluster analysis

visual field to complement these tests showed no deficit. This goes against the hypothesis by Gaudry et al. [60] that perceptual neurovisual impairments could be one of the causes of visual-spatial DCD. It also underlines the problem of the inclusion criteria in the literature samples, which include subjects with visual perception impairment. In Vaivre et al.studies $[11,12,19]$, subjects with visual perception deficits or ADHD were not included, in order to establish pure DCD subtypes. Thus, failure to explore the impact of a visual perception deficit increases the rating of severity of motor impairment, particularly for fine motricity. This failure of distinction between visual perceptual characteristics and visual-motor function, which characterized all the subgroups in Hoare [5] and Macnab et al..s [8] studies, leads to the identification of cognitive deficits for nonverbal IQ measures. This could explain results in the recent study of Asonitou \& Koutsouki [14] highlighting relationships between perception, visuospatial working memory and visuomotor coordination. But there is a limitation to this study, as in all the cluster studies in the literature, relating to the absence of assessment of ADHD symptomatology in the samples. Indeed, it is important not to confuse the deficits explored here with neurovisual disorders of sensory-perceptual origin resulting from brain dysfunctions or lesions (visual zones and pathways) as observed in cerebral motor impairment [60], with DCD. It can be considered that in both visual-perceptual and neurovisual deficits, perception and gestures are bound to be affected. But it is not the same group of DCD children that is diagnosed, because different neuro-anatomical and etiological correlates are involved. It is a differential diagnosis that needs to be clearly established in order to orient care more efficiently. Neurovisual or neuro-perceptual deficits involve the frontal-temporal-parietal occipital pathways, as in the model proposed by Roy [61] in reference to adult apraxia, suggested to explain the etiology of DCD by Dewey \& Kaplan [4]. However, results of Vaivre-Douret et al.[11,12,19] are in line with those of Lundy-Ekman et al.[3], tending, in the VSC group, to demonstrate a semiology of dysfunctional development of subcortical and cerebellar mechanisms secondarily affecting the functioning of the cortical regions $[11,13]$.

The only neurovisual disorder identified $[11,19]$ concerned an ocular-motor disorder in eye pursuit movements, which could reflect a disorder in the lateral-ventral area of the thalamus, as suggested by Tanaka [63] and Ingster-Moati et al. [64], and more recently by Robert et al. [40] who showed a maturation disorder in vertical and horizontal pursuits in DCD children. It should also be noted that no significant deficit in visual exploration strategies were observed, merely a lack of visual exploration without this being hemispatial neglect, more likely to be linked to a disorder in visual pursuit. In addition, no significant deficit in mental planning executive functions were noted (only $5 \%$ ) and the ideomotor representations of mimed praxis were performed by all subjects in symbolic mode (e.g. when brushing teeth with a toothbrush, the brush object is correctly symbolized, and the child does not put his finger in his mouth). There is however a significant correlation in our whole cohort $(12 \%)\left(\mathrm{X}^{2}(1)=3.63 ; \mathrm{p}<0.05\right)$ between the existence of visual perceptual/gnosis deficits and particular perinatal events (nuchal cord, chorionic hematoma, forceps delivery etc). This could point to hypoxic origins for visual-perceptual impairments like co-occurrence for some children because it is not a core symptom of DCD.

In the VSC group, there is a certain number of subjects who presented only the motor visual-spatial component (2D) and not the visual-constructional component (5\% of cases) and this appeared to concern the children with the highest IQ ratings, who may use highlevel mental executive functions, suggested by a score more than 2 SD above the average in mental planning tests of executive functions (the Porteus Labyrinth and the Tower of London). It is consequently possible to consider that certain subjects in the VSC group do not spontaneously use mental imagery to compensate for their perceptualmotor difficulties, reflected by failure on visual-spatial memory and working memory tests. This also supports the hypothesis of the role of the cerebellar region and the basal ganglia reported in the fMRI study by Graydon et al.[65] concerning the development of an internal model of the required task and also learning-related interactions, increasing cerebellar involvement and the role of the putamen in the basal ganglia.

The mixed impairment subgroup (MX): The MX DCD subtype found in Vaivre et al.studies [11,12,19], which combines characteristics of ideomotor DCD (IM) and visual-spatial-constructional DCD (VSC), also evidences significant specific abnormalities $(\mathrm{p}<0.05)$ that are not found in the two "pure" DCD groups, such as impairments in manual dexterity, coordination between upper and lower limbs (global dynamic coordination) (Figure 3, table 2). We have here a set of abnormalities relating to motor coordination that is similar to one of the groups (presenting lower scores) often identified by authors researches on DCD clusters. However, most of these authors only used tests of motor coordination performance (M-ABC) [16]; (BOTMP) [15] without including manual praxis or visual-spatial motor tests. Thus, the inclusion of a complete psychological, neuropsychological, neuro-psychomotor and neurovisual evaluation enabled us to gain a better understanding of all these specific motor coordination abnormalities. In this group there are in particular neurological soft signs or minor neurological dysfunctions suggesting synkinesia and dysdiadochokinesia [20], and disorders in executive functions, and more frequent cognitive auditorymemory deficits and auditory-attention deficits than in the other two subgroups (Figure 3 and Table 2). There have already been various reports on comorbidity between learning disorders and difficulties in the acquisition of coordination, alongside attention deficit with or without hyperactivity, and perception disorders grouped under the term DAMP, with variable levels of the accompanying language difficulties [66]. Rourke [67] suggested a non-verbal dysfunction syndrome combined with greater difficulties in language and social interaction, often described as a hemispheric syndrome of non-verbal dysfunction. In the MX group there are clearly dysfunctions implicating the subcortical-cerebellar network and cortical mechanisms, which can make it difficult to apprehend the functional impact. Nevertheless, since subjects presenting visual perception, attention deficit hyperactivity disorder (ADHD) or language disorders were not included in our study, it is possible to underline the specificity of certain impairments that are markers in this group for comorbidity or co-occurence with other neuropsychological disorders (Figure 3, Table 2). As perception and visual evoked potential impairments did not reach significant levels in this group, it is not possible to explain DCD by a purely neuro-visual cortical effect.

\section{Conclusion}

The profile patterns of three main subtypes of DCD were identified using Hierarchical Ward-based clustering and the k-means algorithm in Vaivre-Douret studies [11,12,19]. Random forest and PLS discriminant analyses were used to reduce the number of relevant features while maximizing the discrimination between the DCD subtypes obtained. Marked impairments with high predictive discriminant power forming two "pure" DCD subgroups were identified: the ideomotor subgroup (IM) characterized by specific impairments $(\mathrm{p}<0.05)$ in digital praxis, imitation of gestures and digital perception; and the visual-spatial/visual-constructional DCD subgroup (VSC) with impairments $(\mathrm{p}<0.05)$ in visual-motor integration, visual-spatial motor structuration, and performances 
Vaivre-Douret L (2019) Specific impairments and predictive markers for developmental coordination disorder subtypes in children: The importance of multidimensional developmental assessments in cluster analysis

on Lego blocks. The Mix subgroup (MX) shares impairments with IM and VSC DCD and has specific impairments $(\mathrm{p}<0.05$, for manual dexterity, coordination between upper and lower limbs), comorbidities of minor neurological dysfunction (dysdiadochokinesis) and cognitive disabilities (executive, auditivo memory, auditivo attention) (Table 2).

The originality and interest of the studies presented here [11,12,19] are that they used multidimensional investigations implicating different assessments of brain functions, exploring psychological, neuropsychological, neurovisual and neuro-psychomotor aspects (with neurological examination of tone and praxis, see NP-Mot battery, table 1) and including a developmental scoring (qualitative and quantitative) of gestures and movements. In the literature the M-ABC battery [16] appears limited to be used as a "gold standard" for the assessment of children with DCD as regards the poor criterion validity for DCD, just as the BOTMP [68-72]. In addition, M-ABC penalizes the performance of children who are unable to attend to or remember specific instructions during the test item [71]. It is not surprising that the most subtypes of DCD found by the authors using the M-ABC or the BOTMP are impossible to understand with neuro-physiological and neuropsychological approaches. Standardiszation with cultural norms of neurodevelopmental assessments such as NP-Mot [18] should be encouraged to be used if we want to understand the nature of impairments and neurophysiological brain mechanisms involved in DCD.

Strict inclusion criteria have to be applied, enabling the specific functions that were significantly affected to be identified, thus distinguishing them from comorbidities or co-occurence [20]. All children met DSM-5 criteria in the studies [11-12, 19] but this is not strictly respected in most of DCD studies because criteria are not verified and a cut off point is not recommended in DSM, so it varies in studies from the 5 th to 15 th percentile.

Indeed, DCD should not be an umbrella diagnosis in the motor spectrum. The evidencing of comorbidity points to other cerebral mechanisms that could explain associated impairments in perception and visual perception, motor execution disorders (pyramidal pathway) $[19,20]$, or executive mental function disorders... etc, thus enabling a differential diagnosis. In the literature it has already been shown that the DAMP concept according to Gillbert [73] and Landgren et al.[74] (deficits in attention, motor control and perception) could be a subgroup of ADHD.

Thus an assessment of visuo-spatial and visuo-motor perception, and the use of a complete standardized developmental assessment battery, including qualitative and quantitative developmental measures of neuromuscular tone, gross and fine motor coordination, with the same tests irrespective of age (e.g. the NP-Mot [18] Table 1) are important for a better identification of variables linked to failure on tests, so as to enable definition of different subtypes of DCD. Qualitative and quantitative measures are important to take into account in the score adjusted on the level of maturation. Furthermore, a global measure of coordination disorder may detect only the postural component or the limb coordination component. Likewise, praxis tests may yield good results from a qualitative viewpoint, but evidence slowness in quantitative terms (timing), or they may be affected by both components. In the same way, tests exploring tone provide information on the organization of tonic maturation and regulation, and on soft signs (synkinesis, hypotonia...). The NP-Mot battery thus provides a more refined evaluation tool to gain understanding of the nature of the disorders observed in relation to maturation. While the M-ABC
[16] may be useful for screening motor impairments, it appears to have limited applicability in diagnosis in 4 to 8 years old [70].

Finally, minor neurological dysfunctions evidenced in the different subtypes of DCD are either neurological soft signs linked to cerebellar control (synkinesis, abnormalities in muscle tone for instance) or to motor control (pyramidal tract) which is an exclusion criterion D in DSM-5. However, it is possible to have a co-occurrence with motor pathway disturbance. [20]. The main motor disorders leading to impairment in motor planning and programming functions (quality of the gesture) or slowness (quantitative aspect) point to a dysfunction of the basal ganglia and the cerebellum.

The findings on specific impairments with high predictive discriminant power provide a definition of different subtypes of DCD and thus enable better orientation of remediation in clinical practice.

We can therefore define DCD as a disturbance in the performance of intentional gestures or movement as a result of dysfunctions in the cerebral planning and programming of movement, upstream from motor execution. These disturbances appear to be specific to sensory and/or visual-spatial motor regulation and integration deficits, upsetting the precision and effectiveness of gestures (spatial and timing parameters). They have to be distinguished from visual perceptual impairments.

Research implementing MRI with fibre tracking and fMRI experimental protocols on DCD groups could provide better understanding of the neuro-anatomical and etiological correlates of DCD subtypes. Indeed, recent studies with structural diffusion magnetic resonance imaging [75-76] corroborate aetiology hypothesis of the three subtypes of Vaivre-Douret et al.[11-12] confirmed by two multivariate statistical models [12-19], showing some alterations of brain microstructure of white matter about corticospinal tract, posterior thalamic radiation and parietal subregion of the corpus callosum. Using functional magnetic resonance imaging in a study of Zwicker et al.[77], it has been demonstrated that there is under-activation in the cerebellar-parietal and cerebellar-prefrontal networks and in brain regions associated with visual-spatial learning. Mean diffusivity of the corticospinal tract and posterior thalamic radiation was lower in children with developmental coordination disorder compared with control children and was significantly correlated with severity of the motor impairment scores on M-ABC-2 [78]. These promising results could explain the Mix group [19] where it is associated sever motor impairment of global coordination often in co-occurrence with motor pathway disturbance [20] and visuo-spatial motor impairment linking to oculomotricity abnormality.

Thus, if M-ABC Test discrimine impairments score on global and fine motricity together, similarly to specific markers of Mix group of DCD (dexterity, global coordination) [12-19] but without knowing the part of gestural planning problem (IM) and visuo-spatial motor impairment (VSC), and the co-occurrence of motor pathway disturbance [20] or from learning disabilities. Then, it is impossible to understand the cause of the impairment, and it remains unclear whether the motor disorder is a DCD on the aetiologic point of view, without to forget the part of cognitive and emotional difficulties to realize some $\mathrm{M}-\mathrm{ABC}$ tasks. This suggests caution when using the M-ABC Test for the purpose of DCD diagnosis in according to Watter et al.., (2008) [70] and Vetnatsanou et al.[71], and it improves to complete investigation of motor disorder with developmental qualitative and quantitative norms and multidimensional assessments of brain function (e.g. attention, executive, visual perception, neurovisual function ) in order to avoid 
Vaivre-Douret L (2019) Specific impairments and predictive markers for developmental coordination disorder subtypes in children:The importance of multidimensional developmental assessments in cluster analysis

false negatives and false positives. A recent study with ASD children [79] showed that the nature of motor impairments is not necessary a diagnosis of DCD considering specific impairments found in Lalanne et al.[19] with high predictive discriminant power markers.

\section{Conflicts of interest}

The authors declare that there are no conflicts of interest.

\section{References}

1. American Psychiatric Association (2013) Diagnostic and statistical manual of mental disorders: DSM-5. 5th edn. Washington, DC: American Psychiatric Association, USA.

2. Lyytinen H, Ahonen T (1988) Developmental motor problems in children: a 6-year longitudinal study. J Clin Exp Neuropsychol 10: 57

3. Lundy-Ekman L, Ivry RB, Keele S, Woollacott M (1991) Timing and force control deficits in clumsy children. $J$ Cogn Neurosci 3: 367-76. [Crossref]

4. Dewey D, Kaplan BJ (1994) Subtyping of developmental motor deficits. Dev Neuropsychol 10: 265-284.

5. Hoare D (1994) Subtypes of developmental coordination disorder. Adapt Phys Activity Q 11:158-169.

6. Miyahara M (1994) Subtypes of students with learning disabilities based upon gross motor functions. Adapt Phys Activity $Q$ 11: 368-382.

7. Wright HC, Sugden DA (1996) The nature of developmental coordination disorder: inter- and intragroup differences. Adapt Phys Activity Q 13: 357-371.

8. Macnab JJ, Miller LT, Polatajko H (2001) The search for subtypes of DCD: Is cluster analysis the answer? Hum Mov Sci 20: 49-72. [Crossref]

9. Green D, Chambers M, Sugden D (2008) Does subtype of developmental coordination disorder count: is there a differential effect on outcome following intervention? Hum Mov Sci 27: 363-382

10. Wilson PH, Ruddock S, Smits-Engelsman B, Polatajko H, Blank R. (2013) Understanding performance deficits in developmental coordination disorder: a metaanalysis of recent research. Dev Med Child Neurol 55: 21.

11. Vaivre-Douret L, Lalanne C, Ingster-Moati I, Boddaert N, Cabrol D, Dufier JL, et al (2011) Subtypes of developmental coordination disorder: Research on their nature and etiology. Dev Neuropsychol 36: 614-643. [Crossref]

12. Lalanne C, Falissard B, Golse B, Vaivre-Douret L (2012) Refining developmental coordination disorder subtyping with multivariate statistical methods. BMC Med Res Methodol 12: 1-14.

13. Vaivre-Douret L (2014) Developmental coordination disorders: state of art. Clin Neurophysiol 44:13-23. [Crossref]

14. Asotinou K, Koutsouki D (2016) Cognitive process-based subtypes of developmental coordination disorder (DCD) Hum Mov Sci 47:121-134.

15. Bruininks H (1978) Bruininks-Oserestky test of motor proficiency. Circles Pines: American Guidance Service.

16. Henderson SE, Sugden DA (1992) Movement Assessment Battery for Children (M-ABC) London, UK: The Psychological Corporation.

17. Beery KE (1982) Revised administration scoring and teaching manual for the development Test of Visual-Motor Integration (VMI) Toronto, Canada: Modern Curriculum Pass.

18. Vaivre-Douret L (2006) Batterie d'evaluation des fonctions neuro-psychomotrices (NPMOT) [Tests battery of developmental Neuro-Psychomotor functions in children (NPMot)]. Paris: Editions du Centre de Psychologie Appliquee-Pearson.

19. Vaivre-Douret L, Lalanne C, Cabrol D, Ingster-Moati I, Falissard B, Golse B, et al. (2011) Identification de critères diagnostiques des sous-types de troubles de l'acquisition de la coordination (TAC) ou dyspraxie developpementale [Identification of diagnostis criteria of developmental coordination disorder (DCD) or developmental dyspraxia subtypes]. Neuropsychiatr Enfance Adolesc 59: 443-453.

20. Vaivre-Douret L, Lalanne C, Golse B. Developmental coordination disorder, an umbrella term for motor impairments in children: nature and co-morbid disorders. Front Psychol.

21. Wilson PH, Pollock N, Kaplan BJ, Law M (1994) Clinical observations of motor and postural skills manual. Tucson, AZ: Therapy Skill Builders.
22. Muttey M, Stirling H, Spalding N (1978) Quick Neurological Screening Test, revised Ed. Northumberland: Ann Arbour Publishers, USA

23. O'Hare A, Khalid S (2002) The association of abnormal cerebellar function in children with developmental coordination disorder and reading difficulties. Dyslexia 8: 234 248. [Crossref]

24. Denckla MB (1985) Revised physical and neurological examination for subtle signs (PANESS) Psychopharmacol Bull 21: 773-800.

25. Touwen BCL (1979) Examination of the child with minor neurological dysfunction Clinics in Developmental Medicine. London, UK: Heinemann Medical Books.

26. Blank R, Barnett AL, Cairney J, Green D, Kirby A, Polatajko H et al. (2019) International clinical practice recommendations on the definition, diagnosis, assessment, intervention, and psychosocial aspects of developmental coordination disorder. Dev Med Child Neurol 61: 242-285. [Crossref]

27. Wilson PH (2005) Practitioner review: Approaches to assessment and treatment of children with DCD: an evaluative review. J Child Psychol and Psychiat 46: 806-823. [Crossref]

28. Nichols PL, abd Chen TC (1981) Minimal brain dysfunction: A prospective study Hillstale, NJ: Lawrence Erlbaum Assoc Incorporated.

29. Khos C (1972) Test des Cubes de Khos Khos's block design test]. Paris, France: Edition du Centre de Psychologie Appliquee-Pearson.

30. Korkman M, Kirk U, Kemp S (2003) Developmental neuropsychological assessmen manual. Paris, France: Editions du Centre de Psychologie Appliquee-Pearson.

31. Douret L, Auzias M (1993) Le developpement de l'organisation motrice et temporospatiale de l'ecriture chez l'enfant [The development of spatial-velocity and motor organization in child handwriting]. ANAE 5: 29-35.

32. Lopez C, Hemimou C, Golse B, Vaivre-Douret L (2018) Developmental dysgraphia is often associated with minor neurological dysfunction in children with developmental coordination disorder (DCD) Clinical Neurophysiology 48: 207-217.

33. Ajuriaguerra (de) J, Auzias M, Denner A (1989) L'ecriture de l'enfant : l'evolution de l'ecriture et ses difficultes [Child handwriting: development of handwriting and impairments]. 3rd ed. Vol. 1. Paris: Delachaux \& Niestle

34. Hamstra-Bletz L, DeBbie J, Den Brinker B (1987) Concise Evaluation Scale for children's handwriting. In : Swets, Zeitlinger, editors. Germany: Lisse.

35. Frostig M (1973) Test de developpement de la perception [A development test of visua perception]. Paris: Editions du Centre de Psychologie Appliquee-Pearson.

36. Odedys (2005) Outils de depistage des Dyslexies, version 2 [Screening tools for dyslexia]. Grenoble: Cogni-Sciences IUFM.

37. Chevrie-Muller C, Plaza M (2001) N-EEL Nouvelles Epreuves pour l'Examen du Langage [New tests for language examination ]. Paris: Editions du Centre de Psychologie Appliquee-Pearson.

38. Ayres AJ (1980) Southern California Sensory Integration Tests Manual - Revised. Los Angeles: Western Psychological Services.

39. Vaivre-Douret L (2002) A more robust predictor of ideomotor dyspraxia study or an alternative scoring method of the Bergès-Lezine's imitation of gestures test. Arch Clin Neuropsychol 17: 37-48.

40. Robert M, Ingster-Moati I, Albuisson E, Cabrol D, Golse B, Vaivre-Douret L, et al. (2014) Vertical and horizontal smooth pursuit eye movements in children with developmental coordination disorder. Dev Med Child Neurol 56: 595-600.

41. Vaivre-Douret L (2004) Precis theorique et pratique du developpement moteur du jeune enfant : normes et dispersions DF-MOT [Theoretical handbook and motor development assessment: DF-MOT standardized scale]. Paris: Editions du Centre de Psychologie Appliquee-Pearson.

42. Geuze R (2005) Motor impairment in DCD and consequences for academic performance and activities of daily living. In: Chambers DASM, editor. Children with developmental coordination disorder. London: Whurr. 3: 19-50.

43. R Core Team. R (2015): A Language and Environment for Statistical Computing. Vienna, Austria: R Foundation for Statistical Computing.

44. Gordon AD (1999) Classification. 2nd ed. London: Chapman and Hall/CRC

45. Tibshirani R, Walther G, Hastie T (2001) Estimating the number of clusters in a data set via the gap statistic. J R Statistical Society, Series B 63: 411-423.

46. Dunn JC (1974) Well separated clusters and fuzzy partitions. J Cybernetics 4: 95-104.

47. Hennig C (2007) Cluster-wise assessment of cluster stability. Comput Stat Data An 52:258-71. 
Vaivre-Douret L (2019) Specific impairments and predictive markers for developmental coordination disorder subtypes in children:The importance of multidimensional developmental assessments in cluster analysis

48. Visser J (2003) Developmental coordination disorder: a review of research on subtypes and comorbidities. Hum Mov Sci 22: 479-493.

49. Nicolson R (2000) Dyslexia and dyspraxia. Commentary 6: 202-214.

50. Vaivre-Douret L (2008) Le point sur la dyspraxie developpementale : symptomatologie et prise en Charge [Developmental dyspraxia : symptomatology and remediation] Contraste 28-29:321-41.

51. Ayres AJ (1972) Sensory integration and learning disorders. Los Angeles: Western Psychological Services.

52. Cermak S (1985) Developmental dyspraxia. In: Roy EA, editor. Neuropsychological studies of apraxia and related disorders. Amsterdam: Elsevier Sciences Publishers, p. $225-48$.

53. Denckla MB (1974) Development of motor coordination in normal children. Dev Med Child Neurol 16: 729-741.

54. Seitz RJ, Roland E, Bohm C, Greitz T, Stone-Elander S, et al. (1990) Motor learning in man: a positron emission tomographic study. Neuroreport 1: 57-60.

55. Mon-Williams MA, Wann JP, Pascal E (1994) Ophthalmic factors in developmental coordination disorder. Adapt Phys Activity $Q$ 11: 170-178.

56. Creavin AL, Lingam R, Northstone k, Williams C (2014) Ophtalmologic abnormalities in children with developmental coordination disorder. Dev Med Child Neurol 5: 164-170.

57. Shoemaker MM, Wees M. van der, Flapper B, Verheij-Janssen N, Scholten-Jaegers S, et al. (2001) Perceptual skills of children with developmental coordination disorder. Hum Mov Sci 20:111-133

58. Van Waelvelde H, De Weerdt W, De Cock P, Smits-Engelsman BCM (2004) Association between visual perceptual deficits and motor deficits in children with developmental coordination disorder. Dev Med Child Neurol 46: 661-666.

59. Milner AD, Goodale MA (1995) The Visual Brain in Action. Oxford: Oxford University Press.

60. Gaudry I, Perez C, Cavezian C, Vilayphonh M, Chokron S (2010) Dyspraxies et troubles neurovisuels [Dyspraxia and neurovisual impairments]. In: Chokron $\mathrm{S}$, Demonet JF, editors. Approche Neuropsychologique des troubles des apprentissages. Marseille: Solal Editeur, p. 297-309.

61. Fazzi E, Signorini GS, Bova MS, La Piana R, Ondei P, Bertone C, et al. (2007) Spectrum of visual disorders in children with cerebral visual impairment. $J$ Child Neurol 22: 294-301.

62. Roy EA (1978) Apraxia: A new look at an old syndrome. J Hum Mov Stud 4: 191-210.

63. Tanaka M (2005) Involvement of the central thalamus in the control of smooth pursuit eye movements. J Neurosci 25: 5866-5876.

64. Ingster-Moati I, Vaivre-Douret L, Delouvrier E, Crochet K, Orssaud C, et al. (2005) Exploration fonctionnelle des voies visuelles sensorielles et oculo-motrices des enfants atteints de dyspraxie developpementale isolee [Functional exploration of visual sensorial pathways and oculomotricity in developmental dyspraxia children]. $J \mathrm{Fr}$ Ophtalmol 28: 107-108.
65. Graydon FX, Friston KJ, Thomas CG, Brooks VB, Menon RS (2005) Learning-related fMRI activation associated with a rotational visuo-motor transformation. Cognitive Brain Res 22: 373-383.

66. Rasmussen P, Gillberg C (2000) Natural outcome of ADHD with developmental coordination disorder at age 22 years: A controlled, longitudinal community-based study. J Am Acad Child Adolesc Psychiatry 39: 1424-431.

67. Rourke BP (1995) Syndrome of non-verbal learning disabilities: Neuro-developmental manifestations. New York, Londres: Guilford Press.

68. Missiuna C, Rivard L, Bartlett D (2006) Exploring assessment tools and the target of intervention for children with developmental coordination disorder. Phys Occup Ther Pediatr 26: 71-89.

69. Slater LM, Hillier SL, Civetta LR (2010) The clinimetric properties of performancebased gross motor tests used for children with developmental coordination disorder: a systematic review. Pediatr Phys Ther 22: 170-179.

70. Water P, Rodger S, Marinac J, Woodyatt G, ZIviani J, et al. (2008) Multidisciplanary assessment of children with developmental coordination disorder: Using the ICF framework to inform assessment. Phys Occup Ther Pediatr 28: 331-352.

71. Venetsanou F, Kambas A, Ellinoudis T, Fatouros I, Giannakidou D, Kourtessis T, et al (2011) Can the movement assessment battery for children-test be the "gold standard" for the motor assessment of children with Developmental Coordination Disorder. Res Dev Disabil 32: 1-10.

72. Crawford SG, Wilson BN, Dewey D (2001) Identifying developmental coordination disorder: consistency between tests. Phys Occup Ther Pediatr 20: 29-50.

73. Gillberg C (1998) Hyperactivity, inattention and motor control problems - prevalence, comorbidity and background factors. Folia Phoniatr Logop 50: 107-117.

74. Landgren M, Pettersson R, Kjellman B, Gillbert C (1996) ADHD, DAMP and other neurodevelopmental/psychiatric disorders in 6-year-old children: epidemiology and comorbidity. Dev Med Child Neurol 38: 891-906.

75. Debrabant J, Vingerhoets G, Van Waelvelde H, Leemans A, Taymans T, Gaeyenberghs $\mathrm{K}$, et al. (2016) Brain connectomics of visual-motor deficits in children with developmental coordination disorder. J Pediatr 169: 21-27.

76. Zwicker JG, Missiuna C, Harris SR, Boyd LA (2012) Developmental coordination disorder: a pilot diffusion tensor imaging study. Pediatr Neurol 46: 162-167.

77. Zwicker JG, Missiuna C, Harris SR, Boyd LA (2011) Brain activation associated with motor skill practice in children with developmental coordination disorder: an fMRI study. Int J Dev Neuroscience 29: 145-152.

78. Henderson SE, Sugden DA (2007) Movement Assessment Battery for Children (M-ABC-2) $2^{\text {nd }}$ edn. London, UK: Harcourt Assessment.

79. Paquet A, Olliac B, Golse B, Vaivre-Douret L (2019) Nature of motor impairments in Autism Spectrum Disorder: a comparison with Developemental Coordination Disorder $J$ of $\mathrm{Cl}$ and Exp neuropsy 41: 1-14.

Copyright: (C)2019 Vaivre-Douret L. This is an open-access article distributed under the terms of the Creative Commons Attribution License, which permits unrestricted use, distribution, and reproduction in any medium, provided the original author and source are credited. 